\title{
CARACTERIZAÇÃO FENOTÍPICA DE OVELHAS DOS HUMEDALES DE ITE, SUL DO PERU: DADOS PRELIMINARES
}

\author{
PHENOTYPIC CHARACTERIZATION OF EWES FROM ITE WETLANDS, SOUTHERN \\ PERU: PRELIMINARDATA
}

\author{
Montesinos, I.S. ${ }^{1 *}$, Silva, M.C. ${ }^{1}$, Lopes, F.B. ${ }^{1}$, Fioravanti, M.C.S. ${ }^{1}$, McManus, C.M. ${ }^{2}$ \\ e Sereno, J.R.B. ${ }^{3}$
}

\begin{abstract}
1Programa de Pós-Graduação em Ciência Animal. Escola de Veterinária e Zootecnia. Universidade Federal de Goiás. Goiânia-GO. Brasil. *ivansm.vet@gmail.com

${ }^{2}$ Departamento de Zootecnia. Universidade Federal de Rio Grande do Sul. Porto Alegre-RS. Brasil.

${ }^{3}$ Empresa Brasileira de Pesquisa Agropecuária. Embrapa Cerrados. Planaltina-DF. Brasil.
\end{abstract}

\section{PalaVRas ChaVE ADICIONAIS}

Conservação. Ovinocultura. Raças locais.

\section{RESUMO}

A ovinocultura é uma importante atividade socioeconômica no Peru, sendo uma das principais fontes de renda para muitas famílias rurais. O objetivo do presente estudo foi caracterizar o fenótipo de ovelhas adultas, procedentes de rebanhos locais criados nos Humedales de Ite, litoral sul do Peru. Estes animais estavam sob sistema semi-extensivo, em pastagens nativas. Treze medidas morfométricas foram mensuradas e analisadas usando os procedimentos MEANS, CORR, GLM, DISCRIM e CLUSTER do programa estatístico SAS. Três medidas morfológicas também foram registradas e três índices zoométricos calculados. Os resultados indicaram que estas ovelhas são menores do que as raças especializadas, mas possuem tendência para aptidão de corte e diferentes tamanhos corporais, mostrando variabilidade de biótipos entre rebanhos de acordo ao manejo, localização geográfica e aparente miscigenação racial.

\section{SUMMARY}

Sheep farming is an important socioeconomic activity in Peru, being one of the main sources of income for many rural families. The objective of the present study was to characterize the phenotype of adult ewes, from local flocks farmed in the Ite Wetlands, southern coast of Peru. These animals were reared in a semi extensive system, on native

\author{
AdDitional KEYWORDS \\ Conservation. Local breeds. Sheep breeding.
}

pastures. Thirteen morphometric traits were measured and analyzed using MEANS, CORR, GLM, DISCRIM and CLUSTER procedures of the statistical program SAS. Three morphologic traits were also registered and three zoometric indices calculated. The results indicated that these ewes are smaller than specialized breeds, but had tendency for meat production and different body sizes, showing variability of biotypes between flocks, according to the management, geographical location and apparent racial miscegenation.

\section{INTRODUÇÃO}

No Peru, a ovinocultura é uma importante atividade pecuária, principalmente para os pequenos agricultores, os quais representam em média $80 \%$ dos produtores rurais no país. O rebanho nacional está constituído por 15 milhões de ovinos, dos quais cerca de $60 \%$ são crioulos (INIA, 2003).

O termo crioulo ou local é utilizado para denominar os grupos genéticos que descendem das raças introduzidas pelos colonizadores europeus no século XVI, e que ao longo do tempo evoluíram, adaptando-se a diversas condições sanitárias, de clima e manejo encontradas no novo continente (Egito et al., 2002). 
Os ovinos crioulos no Peru segundo Alencastre e Gómez (2005), descendem de troncos ancestrais trazidos da península Ibérica (Merino, Entrefino, Churro e Ibérico) e atualmente encontram-se mais difundidos que as raças especializadas, devido ao baixo custo de aquisição e manutenção, além de ter maior resistência a situações adversas, diminuindo a necessidade de grande investimento por parte dos criadores.

Estes animais fornecem carne, lã, couro e esterco para os proprietários. Proporcionam também incrementos econômicos em tempos de necessidade, já que podem ser vendidos em qualquer época do ano (Fulcrand, 2005).

No Peru, a ovinocultura se desenvolve em sua maioria na região serrana $(96,2 \%)$, seguida da costeira $(3,2 \%)$ e selva amazônica $(0,4 \%)$. A região costeira caracteriza-se pela pouca precipitação pluvial, requerendo sistemas de criação intensivos ou semiextensivos que utilizam geralmente subprodutos da horticultura como fonte alimentar. Na região serrana e amazônica, as pastagens naturais são decorrentes das estações chuvosas, justificando-se assim o fato da maioria dos sistemas de criação ser do tipo extensivo (INIA, 2003).

A partir do século XX, o ovino crioulo peruano foi submetido a cruzamentos absorventes com raças especializadas, como a Merino e Corriedale, na tentativa de melhorar os rebanhos locais. Em 1921, importaram-se da Inglaterra ovinos Southdown, Hampshire Down, Suffolk e Romney Marsh, apoiados na sua difusão pelo governo mediante o Banco de Fomento Agropecuário. Com o advento da reforma agrária (1969-1979) as importações cessaram e voltaram a ocorrer na década de 1980, com animais da Nova Zelândia, Argentina e Estados Unidos, destacando a raça Hampshire Down que foi introduzida na região costeira de todo Peru (Flores, 2008).

Explorar os ovinos crioulos nunca foi uma prioridade nos governos deste país, o que pode ser verificado pela falta de fomen- to, pesquisas e uma política pública específica para este tema. Tendo como conseqüência a baixa produtividade desses animais (Fulcrand, 2005).

Os eventos ocorridos através do tempo na ovinocultura do Peru foram refletidos ao estudar rebanhos localizados na região sul do país, no município rural de Ite, onde uma associação de ovinocaprinocultores, formada por 19 famílias de origem aymara, pratica a criação semi-extensiva de ruminantes menores com finalidade de produzir carne e leite, uma atividade complementar ao orçamento familiar.

Na faixa litorânea deste município existe uma zona chamada Humedales, que possui 12 km de extensão e 2000 ha de superfície. Segundo Vizcarra (2008), é um ecossistema constituído por flora e fauna nativa. Nos setores descobertos crescem plantas como a Distichlis spicata, Cynodon dactylon e Bacopa monnieri, enquanto nos setores alagados além das espécies já mencionadas, existem a Typha angustifólia e Scirpus californicus. Esta vegetação é aproveitada pelos ovinocaprinocultores na produção animal, uma vez que a maioria deles não dispõe de terras próprias para desenvolver agricultura. Diferente de outros habitantes do município, os quais possuem chácaras nos setores de Alfarillo, Pampa Baja ou Pampa Alta, dedicando-se a atividades como lavoura e pecuária.

Os ovinos são liberados em pastos nativos pela manhã e recolhidos ao curral no final da tarde. Geralmente os responsáveis por este trabalho são as mulheres, crianças e idosos, uma vez que os homens realizam atividades paralelas para garantir o sustento familiar. Esta produção animal é de subsistência e extrativista, pelos baixos recursos econômicos dos criadores, que utilizam só os pastos naturais como alimento para os rebanhos, sem nenhum tipo de forragem ou ração suplementar, além da inexistência na maioria dos casos de um calendário profilático-sanitário e de manejo, devido a pouca assistência técnica no local. 
Sabe-se também da existência de animais com diferentes graus de miscigenação Hampshire Down, os quais são explorados nos setores das chácaras pelos agricultores de Ite. Estes ovinos representam uma ameaça para os rebanhos locais dos Humedales, precioso material genético obtido ao longo do tempo pela seleção natural (Mariante e Cavalcante, 2006). No último censo registraram-se 972 ovinos nos Humedales e 1971 entre os setores Alfarillo, Pampa Baja e Pampa Alta (Robles, 2009).

O ovino crioulo ainda não foi reconhecido como raça pelo Ministério da Agricultura do Peru. Entretanto, constata-se a necessidade de maiores estudos por parte da comunidade científica, buscando o reconhecimento racial deste recurso genético animal, adaptado por séculos ao ambiente econômico, social e ecológico das diferentes regiões no país.

Considerando a escassez de informações sobre estes animais no sul do Peru, é que se resolveu iniciar esta pesquisa. Desse modo, objetivou-se caracterizar fenotipicamente ovelhas adultas, procedentes de rebanhos locais criados nos Humedales de Ite, e assim contribuir no futuro desenvolvimento de um programa de conservação para o ovino crioulo nesta região.

\section{MATERIALE MÉTODOS}

O trabalho foi realizado em janeiro de 2011, no município rural de Ite, região de Tacna, província de Jorge Basadre Grohmann. Latitude sul 17 $50^{\prime} 27^{\prime \prime}$ e Longitude oeste $70^{\circ} 57^{\prime} 47^{\prime \prime}$ (figura 1).

Foram avaliadas 100 ovelhas adultas procedentes de 4 rebanhos (25 fêmeas por rebanho), cujo critério de escolha dentro dessa categoria, foi a troca dos quatro pares de dentes incisivos inferiores (figura 2).

As medidas morfométricas foram coletadas com auxilio de fita métrica e bastão zootécnico, enquanto as morfológicas estimadas visualmente, ambas as mensurações conforme metodologia utilizada por Oliveira et al. (2007). Variáveis morfométricas: largura da cabeça (LCa), comprimento da cabeça $(\mathrm{CCa})$, perímetro da canela $(\mathrm{PCa})$, altura da cernelha (ACe), diâmetro dorsoesternal (DDe), perímetro torácico (PTo), diâmetro entre costelas ou bi-costal (DCo), comprimento corporal (CCo), altura da garupa (AGa), largura da garupa (LGa), comprimento da garupa (CGa), largura entre ancas (LAn) e largura entre ísquios (LIs). Variáveis morfológicas: tamanho de orelhas (TO), perfil cefálico (PC) e condição corpo$\operatorname{ral}(\mathrm{CC})$.

Também foram calculados segundo Bravo e Sepúlveda (2010), os seguintes índices zoométricos: corporal (CCo*100/PTo), profundidade (DDe*100/ACe) e pélvico (LGa*100/CGa).

Para determinar valores médios, coeficientes de variação, desvios padrão, estimativas de correlação e testar efeito do rebanho sobre as medidas morfométricas, utilizaram-se os procedimentos MEANS, CORR, GLM, DISCRIM e CLUSTER do programa computacional Statistical Analysis System (SAS, 2001). Os índices zoométricos

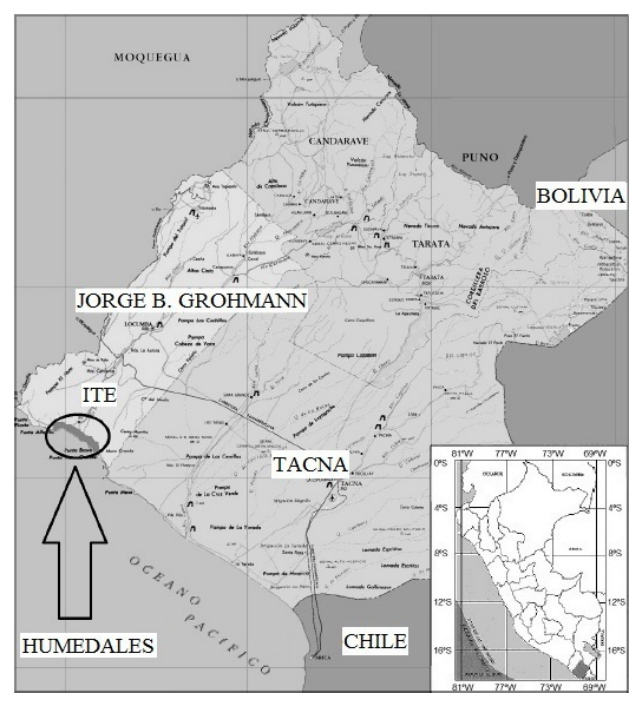

Figura 1. Região de Tacna, sul do Peru. (Region of Tacna, southern Peru). 


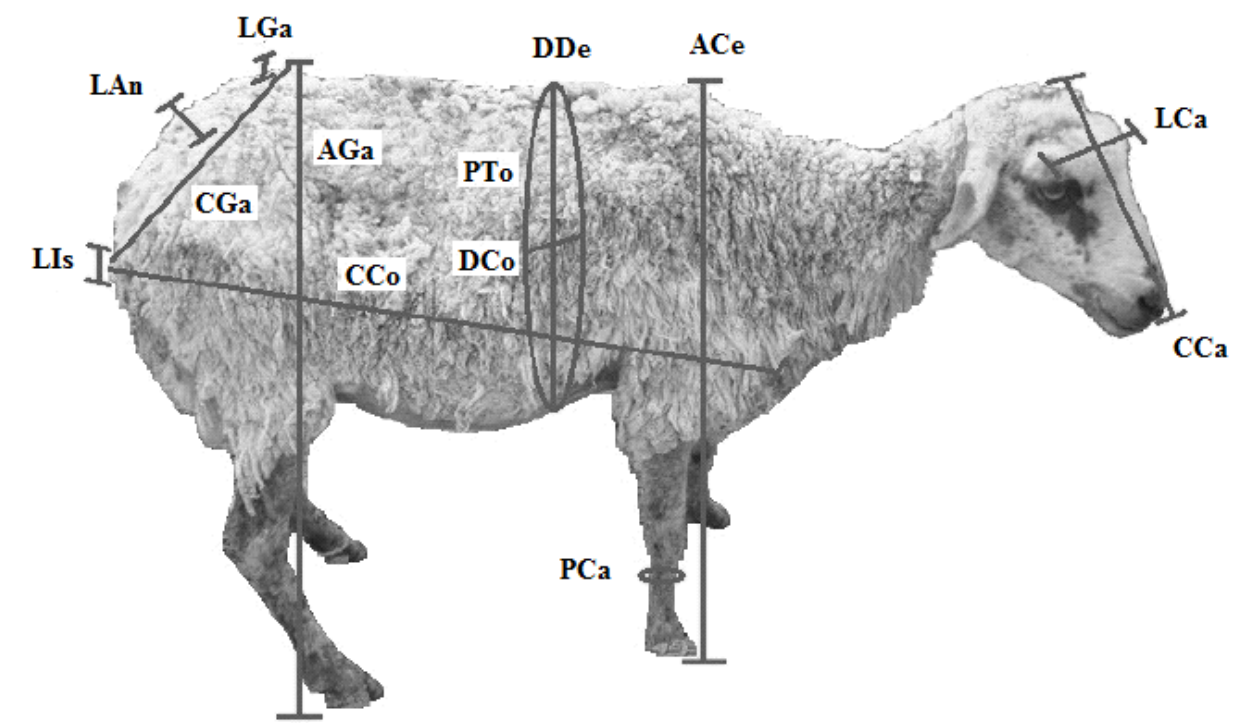

\section{LCa Largura da Cabeça \\ CCa Comprimento da Cabeça \\ PCa Perímetro da Canela \\ ACe Altura da Cernelha \\ DDe Diâmetro Dorso-esternal}

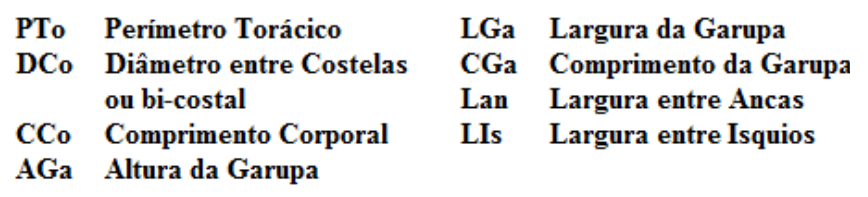

Figura 2. Medidas morfométricas avaliadas em ovelhas adultas criadas nos Humedales de Ite. (Morphometric measurements assessed in adult ewes farmed in the Ite Wetlands).

e freqüências relativas das medidas morfológicas se calcularam em planilhas de Excel. A diferença estatística entre as médias das medidas morfométricas e índices zoométricos dos quatro rebanhos foi testada pelo teste Duncan, ao nível de significância de $5 \%$.

\section{RESULTADOSEDISCUSÃO}

A ovinocultura desenvolvida nos Humedales de Ite é importante para manutenção das famílias que praticam esta atividade, e que até o momento não foi objeto de investigação, tornando-se um trabalho pioneiro com estes recursos genéticos locais.

Inicialmente esperava-se encontrar animais com fenótipo similar, entretanto, à medida que as coletas de campo evoluíram encontraram-se diferenças entre rebanhos, percebidas visualmente e por meio do discurso dos criadores. Vale salientar que este saber zootécnico dos proprietários é ressaltado na etnozootecnia (Alves et al., 2010), sendo importante e oportuno nesta pesquisa.

As divergências entre os rebanhos foram conferidas na análise dos dados, obtendose variabilidade de biótipos segundo o manejo praticado, localização geográfica e possíveis graus de miscigenação racial.

Estes resultados preliminares foram relevantes, já que serviram para conhecer a situação da ovinocultura local, possibilitando delinear futuros estudos de caracterização fenotípica, genotípica e atividades subseqüentes que visem a 
conservação do ovino crioulo no sul do Peru.

A primeira etapa foi a coleta das medidas morfométricas e morfológicas a campo, realizada geralmente no primeiro horário da manha, antes dos animais serem liberados dos currais. Foi possível mensurar 100 ovelhas adultas pertencentes a quatro rebanhos, os quais estavam localizados nas zonas norte (A e B) e sul (C e D) dos Humedales, sabendo que em 2008 foi estimada uma população adulta de 546 fêmeas e 85 machos (Robles, 2009).

Os rebanhos A e B foram criados em zonas com maior oferta de pastos, devido às águas perenes desse setor, ao contrário dos rebanhos $\mathrm{C}$ e $\mathrm{D}$, que possuíam menor oferta de água e vegetação. Os proprietários do rebanho B moravam próximos aos currais, tendo maior contato e dedicação no manejo reprodutivo e sanitário dos animais, diferente dos rebanhos $\mathrm{A}, \mathrm{C}$ e $\mathrm{D}$, cujos proprietários moravam na zona urbana de Ite, visitando os criatórios só pela manha e no final da tarde.

Após coleta dos dados estes foram processados, obtendo-se inicialmente resultados da análise descritiva das características morfométricas (tabela I), que revelou médias inferiores às encontradas em raças especializadas como a Dorper, Suffolk e Bergamácia, estudadas por Carneiro et al. (2010).

Segundo Santana et al. (2001), valores inferiores de CCo, PTo, ACe e AGa podem indicar que os ovinos crioulos do Peru possuem menor rendimento de carcaça, o que é esperado em criações de subsistência, com menor aporte tecnológico e qualidade alimentar, retratando a condição socioeconômica dos proprietários.

Por outro lado, os valores de CGa e LAn são superiores aos encontrados por aqueles autores em ovinos Santa Inês e Crioulos Uruguaios, assim como raças especializadas Suffolk e Texel. Medidas maiores da estrutura pélvica podem ser indicativas de facilidade de parto, característica própria de animais sujeitos a pressões de seleção natural, como os rebanhos crioulos (Primo, 1992). Esta característica é apontada pelos criadores como importante, para diminuir perdas nas épocas de parição pelo ataque de raposas (Pseudalopex griseus) e aves de rapina (Geranoaetus melanoleucus, $\mathrm{Ca}$ thartes aura), predadores freqüentes nos Humedales de Ite (Vizcarra, 2008).

PTo e CCo tiveram os maiores valores de desvio padrão entre as variáveis morfométricas analisadas, corroborando estimativas evidenciadas por Oliveira et al. (2007) na raça Texel, e Silva (2011) na raça Crioula Lanada. Sabe-se que estas duas variáveis podem ser utilizadas em estudos de índice corporal (McManus et al., 2008).

No coeficiente de variação $(\mathrm{CV})$, LIs $(10,98)$ obteve a maior porcentagem seguido de LGa $(9,54)$ e DCo $(9,08)$. Estas medidas indicam a largura do animal, correspondendo LIs e LGa à cavidade pélvica e DCo à cavidade torácica, regiões anatômicas que ao obter $\mathrm{CV}$ altos evidenciaram

Tabela I. Média, desvio padrão (DP) e coeficiente de variação (CV) para medidas morfométricas (cm) de ovelhas adultas criadas nos Humedales de Ite. (Mean, standard deviation (DP) and coefficient of variation (CV) for morphometric measurements $(\mathrm{cm})$ of adult ewes farmed in the Ite Wetlands).

\begin{tabular}{lccc}
\hline & Média & DP & CV (\%) \\
\hline CCa & 23,09 & 1,78 & 5,35 \\
LCa & 13,49 & 1,13 & 8,02 \\
CCo & 75,94 & 5,22 & 4,34 \\
ACe & 62,15 & 2,23 & 3,46 \\
PTo & 88,20 & 7,18 & 4,98 \\
PCa & 7,58 & 0,67 & 7,71 \\
DCo & 28,35 & 3,73 & 9,08 \\
DDe & 30,75 & 2,72 & 7,15 \\
AGa & 64,41 & 2,61 & 4,06 \\
CGa & 23,92 & 1,94 & 7,27 \\
LGa & 20,12 & 2,28 & 9,54 \\
LAn & 26,78 & 1,92 & 7,02 \\
LIs & 8,46 & 0,95 & 10,98 \\
& & & \\
\hline
\end{tabular}


novamente variabilidade no tamanho do corpo. Silva (2011) reportou valores de CV superiores na maioria das medidas morfométricas da raça Crioula Lanada, o que sugere maior heterogeneidade fenotípica, talvez por tratar-se de rebanhos mais distantes entre si, sob diferentes condições edafoclimáticas na região sul do Brasil.

O rebanho B apresentou médias superiores, em relação aos rebanhos $\mathrm{A}, \mathrm{C}$ e D para CCo $(82,32)$, PTo $(97,68)$, LGa $(21,96)$ e $\mathrm{CCa}(25,04)$ (tabela II).

Isto poderia indicar melhor manejo e oferta de pastos a que o rebanho $\mathrm{B}$ foi submetido, assim como um genótipo superior para desempenho zootécnico. Tais fatores em conjunto teriam permitido a criação de animais maiores.

Cabe ressaltar que se encontraram muitos exemplares deste criatório com uma coloração negra na cara e canelas (figura 3), indicando possíveis cruzas com ovinos descendentes da raça Hampshire Down, os

Tabela II. Médias das medidas morfométricas (cm) em quatro rebanhos de ovelhas adultas criadas nos Humedales de Ite. (Means of morphometric measurements $(\mathrm{cm})$ in four flocks of adult ewes farmed in the Ite Wetlands).

\begin{tabular}{lcccc}
\hline & A & B & C & D \\
\hline CCa & $22,76^{\mathrm{b}}$ & $25,04^{\mathrm{a}}$ & $21,44^{\mathrm{c}}$ & $23,12^{\mathrm{b}}$ \\
LCa & $13,84^{\mathrm{a}}$ & $13,64^{\mathrm{a}}$ & $12,84^{\mathrm{b}}$ & $13,64^{\mathrm{a}}$ \\
CCo & $74,80^{\mathrm{b}}$ & $82,32^{\mathrm{a}}$ & $71,04^{\mathrm{c}}$ & $75,60^{\mathrm{b}}$ \\
ACe & $62,60^{\mathrm{a}}$ & $62,76^{\mathrm{a}}$ & $60,96^{\mathrm{b}}$ & $62,28^{\mathrm{a}}$ \\
PTo & $87,60^{\mathrm{b}}$ & $97,68^{\mathrm{a}}$ & $83,24^{\mathrm{c}}$ & $84,28^{\mathrm{c}}$ \\
PCa & $7,72^{\mathrm{ab}}$ & $8,04^{\mathrm{a}}$ & $7,12^{\mathrm{c}}$ & $7,44^{\mathrm{bc}}$ \\
DCo & $30,08^{\mathrm{a}}$ & $31,40^{\mathrm{a}}$ & $24,24^{\mathrm{c}}$ & $27,68^{\mathrm{b}}$ \\
DDe & $31,72^{\mathrm{a}}$ & $32,72^{\mathrm{a}}$ & $28,36^{\mathrm{c}}$ & $30,20^{\mathrm{b}}$ \\
AGa & $64,72^{\mathrm{a}}$ & $64,56^{\mathrm{a}}$ & $63,76^{\mathrm{a}}$ & $64,60^{\mathrm{a}}$ \\
CGa & $24,36^{\mathrm{ab}}$ & $25,00^{\mathrm{a}}$ & $22,52^{\mathrm{c}}$ & $23,80^{\mathrm{b}}$ \\
LGa & $19,96^{\mathrm{b}}$ & $21,96^{\mathrm{a}}$ & $18,36^{\mathrm{c}}$ & $20,20^{\mathrm{b}}$ \\
LAn & $26,60^{\mathrm{ab}}$ & $27,64^{\mathrm{a}}$ & $26,56^{\mathrm{ab}}$ & $26,32^{\mathrm{b}}$ \\
LIs & $8,52^{\mathrm{ab}}$ & $8,48^{\mathrm{ab}}$ & $8,08^{\mathrm{b}}$ & $8,76^{\mathrm{a}}$ \\
\hline
\end{tabular}

abcLetras diferentes na mesma linha indicam diferença significativa $(p<0,05)$ pelo teste Duncan.

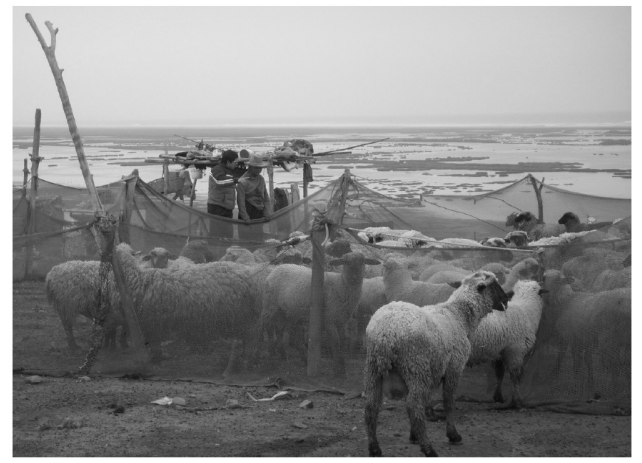

Figura 3. Rebanho dos Humedales de Ite mostrando possível miscigenação com a raça Hampshire Down. (Flock from Ite Wetlands showing possible miscegenation with the breed Hampshire Down).

quais habitam nos setores das chácaras. Esta suposta miscigenação do rebanho B aparentemente influiu no maior tamanho corporal, assim como relata Chacón et al. (2011) em caprinos mestiços da raça Anglonubiana.

Com exceção de PTo $(83,24), \mathrm{PCa}(7,12)$, AGa $(63,76)$, LAn $(26,56)$ e LIs $(8,08)$, as medidas morfométricas do rebanho $\mathrm{C}$ foram menores do que rebanhos A, B e D ( $\mathrm{p}<0,05)$. Pressões de seleção em condições adversas podem ter interferido neste resultado (Bianchini et al., 2006), tendo em vista que o sistema de manejo e oferta dos pastos do criatório C são piores. Outra explicação pelo menor tamanho é o fato de que aparentemente estes animais possuíam menos indícios de miscigenação com raça exótica, revelado pelo fenótipo similar aos ovinos crioulos da região serrana do Peru, mencionados por Fulcrand (2005), possuindo parte do rebanho $\mathrm{C}$ a mesma coloração e tipo de perfil cefálico.

$\mathrm{Na}$ figura 4, observam-se as diferenças fenotípicas visíveis encontradas entre os quatro rebanhos (A, B, C e D), as quais não eram esperadas antes da coleta de campo. Esta variabilidade pode ser devida à origem, condições ambientais, assim como prefe- 


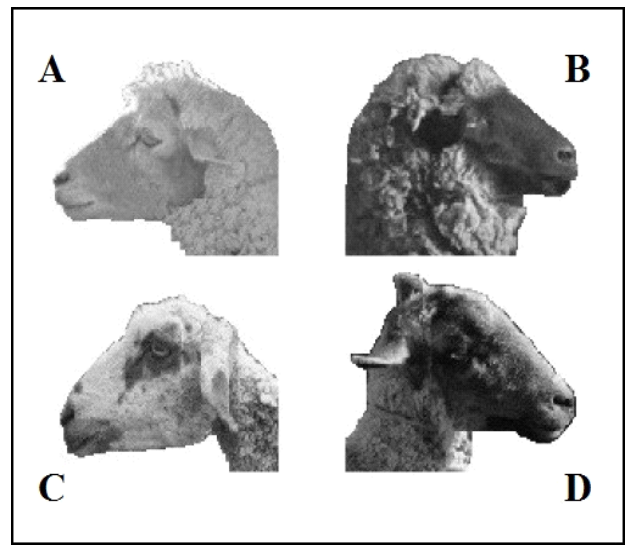

Figura 4. Fenótipos particulares de cada rebanho. (Particular phenotypes of each flock).

rência dos criadores por um tipo de animal (Miserani et al., 2002; Riva et al., 2004).

A diferença das medidas morfométricas entre rebanhos também foi verificada pela análise de variância, na qual o efeito do rebanho foi significativo $(\mathrm{p}<0,05)$ sobre a maioria das variáveis estudadas, o que já foi reportado em outras pesquisas (Dossa et al., 2007; Carneiro et al., 2010; Silva, 2011).

Na tabela III, segundo o índice corporal (ICO) quase todos os animais eram brevilíneos (ICO<86\%) a exceção do rebanho D (longilíneo). Fato que demonstra a diversidade que existe na espécie ovina, seja entre animais da mesma raça ou raças diferentes (Pacheco e Quirino, 2008).

Menores valores de ICO revelam aparência retangular, típica de animais de corte, tendo o rebanho $\mathrm{B}$ o biótipo mais característico (Salako, 2006), e, além disso, conseguiu valores superiores no índice de profundidade (IPRF), o que indica extremidades curtas, apropriadas para pastejo e engorda, corroborando assim resultados anteriores. $\mathrm{O}$ rebanho $\mathrm{C}$ obteve valores inferiores no IPRF, os quais correspondem a animais ligeiros, de pernas compridas para longas caminhadas e velocidade (Álvarez et $a l ., 2000)$, revelando que são ovelhas menos especializadas, mas com adaptação ao ambiente rústico em que habitam.

O índice pélvico (IPE) está associado à estrutura da garupa e reprodução das fêmeas. Os percentuais superiores do rebanho B indicam uma garupa quadrangular e ampla, com predisposição para facilidade de parto (Rodríguez et al., 1990), enquanto os valores do rebanho $\mathrm{C}$ revelam uma menor amplitude a ser melhorada.

Esta heterogeneidade dos rebanhos deve ser vista como um recurso genético que gera oportunidades, já que permite criar grupos e explorá-los, intensificando a eficiência na produção animal (Cartwright, 1979).

Na tabela IV se observa que no tamanho de orelhas (TO), os quatro criatórios apresentaram mais de $50 \%$ de animais com orelhas medianas. Além disso, nos rebanhos C $(44 \%)$ e D $(36 \%)$ se encontraram valores superiores para orelhas grandes em comparação aos outros grupos.

Tabela III. Índices zoométricos em quatro rebanhos de ovelhas adultas criadas nos Humedales de Ite. (Zoometric indices in four flocks of adult ewes farmed in the Ite Wetlands).

\begin{tabular}{lcccccccccccc}
\hline & \multicolumn{3}{c}{ Rebanho A } & \multicolumn{3}{c}{ Rebanho B } & \multicolumn{3}{c}{ Rebanho C } & \multicolumn{3}{c}{ Rebanho D } \\
& Média & DP & CV & Média & DP & CV & Média & DP & CV & Média & DP & CV \\
\hline ICO & $85,48^{\mathrm{b}}$ & 3,13 & 3,66 & $84,42^{\mathrm{b}}$ & 4,01 & 4,75 & $85,48^{\mathrm{b}}$ & 5,05 & 5,91 & $89,79^{\mathrm{a}}$ & 3,82 & 4,25 \\
IPRF & $50,65^{\mathrm{a}}$ & 2,99 & 5,90 & $52,18^{\mathrm{a}}$ & 3,69 & 7,07 & $46,52^{\mathrm{c}}$ & 3,72 & 8,00 & $48,49^{\mathrm{b}}$ & 2,18 & 4,50 \\
IPE & $82,69^{\mathrm{ab}}$ & 11,30 & 13,67 & $88,09^{\mathrm{a}}$ & 8,90 & 10,10 & $81,76^{\mathrm{b}}$ & 11,14 & 13,63 & $85,21^{\mathrm{ab}}$ & 7,06 & 8,29 \\
\hline
\end{tabular}

abcLetras diferentes na mesma linha indicam diferença significativa $(p<0,05)$ pelo teste Duncan. 
MONTESINOS, SILVA, LOPES, FIORAVANTI, MCMANUS E SERENO

Tabela IV. Freqüências absolutas (FA) e relativas $(F R)$ de medidas morfológicas em quatro rebanhos de ovelhas adultas criadas nos Humedales de Ite. (Absolut and relative frequencies of morphologic measurements in four flocks of adult ewes farmed in the Ite Wetlands).

\begin{tabular}{|c|c|c|c|c|c|c|c|c|}
\hline & \multicolumn{2}{|c|}{ Rebanho A } & \multicolumn{2}{|c|}{ Rebanho B } & \multicolumn{2}{|c|}{ Rebanho C } & \multicolumn{2}{|c|}{ Rebanho D } \\
\hline & $\mathrm{FA}(\mathrm{n})$ & $\mathrm{FR} \%$ & FA (n) & $\mathrm{FR} \%$ & FA (n) & $\mathrm{FR} \%$ & $\mathrm{FA}(\mathrm{n})$ & $\mathrm{FR} \%$ \\
\hline \multicolumn{9}{|l|}{ TO } \\
\hline Pequenas & 4 & 16,00 & 6 & 24,00 & 1 & 4,00 & 3 & 12,00 \\
\hline Medianas & 16 & 64.00 & 13 & 52,00 & 13 & 52,00 & 13 & 52,00 \\
\hline Grandes & 5 & 20,00 & 6 & 24,00 & 11 & 44,00 & 9 & 36,00 \\
\hline \multicolumn{9}{|l|}{$\mathrm{PC}$} \\
\hline Reto & 20 & 80,00 & 20 & 80,00 & 12 & 48,00 & 13 & 52,00 \\
\hline Convexo & 5 & 20,00 & 5 & 20,00 & 13 & 52,00 & 12 & 48,00 \\
\hline \multicolumn{9}{|l|}{$\mathrm{CC}$} \\
\hline Magra & 5 & 20,00 & 3 & 12,00 & 4 & 16,00 & 1 & 4,00 \\
\hline Regular & 18 & 72,00 & 14 & 56,00 & 18 & 72,00 & 22 & 88,00 \\
\hline Gorda & 2 & 8,00 & 8 & 32,00 & 3 & 12,00 & 2 & 8,00 \\
\hline
\end{tabular}

No perfil cefálico (PC) os criatórios A e B obtiveram $80 \%$ de animais com perfil reto, enquanto os rebanhos C (48\%) e D $(52 \%)$ valores menores, no entanto altas porcentagens para PC convexo (C $52 \%$; D $48 \%$ ).

As freqüências relativas (FR) obtidas pelos grupos C e D para orelhas grandes e perfil cefálico convexo evidenciaram similaridade étnica entre esses rebanhos, e diferenças com os criatórios A e B da zona norte (Rodríguez et al., 2003). Talvez por estarem os rebanhos $\mathrm{C}$ e $\mathrm{D}$ mais próximos, com trocas genéticas freqüentes e de maior intensidade (Herrera et al., 1996).

Estes resultados mostraram novamente a variabilidade entre os rebanhos, só que nesta oportunidade pode ser mais devido a fatores genéticos, do que ambientais e de manejo.

A maioria dos animais apresentou CC regular, com FR superiores a $55 \%$ em cada rebanho. Entretanto, o criatório B mostrou maior número de ovelhas com CC gorda (32 $\%$ ), o que revelou melhor oferta e aproveitamento dos pastos, sabendo que este rebanho possui possivelmente o melhor potencial para aptidão de corte em comparação aos outros grupos (A, C e D).

Na tabela $\mathbf{V}$, a análise discriminante mostra que o rebanho $\mathrm{B}$, localizado na zona norte dos Humedales, apresentou melhor porcentual de classificação (92\%), seguido do rebanho $\mathrm{C}$ da zona sul $(88 \%)$ e os rebanhos A e D $(72 \%)$.

Ao nível morfométrico os rebanhos $\mathrm{B}$ e C são mais característicos e homogêneos do que A e D. Possivelmente porque o criatório B possui maior grau de miscigenação com a raça Hampshire Down, somado a isso melhor manejo e pastagens, enquanto o C é típico dos ovinos crioulos do Peru, além de ter manejo e pastos de menor qualidade. Cabe ressaltar que os rebanhos $\mathrm{A}$ e $\mathrm{D}$ podem ter

Tabela V. Percentual de ovelhas classificadas em cada procedência geográfica dos Humedales de Ite. (Percentage of ewes classified in each geographical origin from Ite Wetlands).

\begin{tabular}{lcccc}
\hline $\begin{array}{l}\text { Procedência } \\
\text { (rebanho) }\end{array}$ & A & B & C & D \\
\hline A & 72 & 0 & 8 & 20 \\
B & 0 & 92 & 0 & 8 \\
C & 0 & 0 & 88 & 12 \\
D & 16 & 4 & 8 & 72 \\
Taxa de erro & 28 & 8 & 12 & 28 \\
\hline
\end{tabular}


graus de miscigenação intermediários pelos fenótipos apresentados (figura 5).

Na tabela VI, observa-se que a maioria das estimativas de correlação de Pearson foram significativas $(\mathrm{p}<0,05)$. A estimativa mais forte foi entre CCo e PTo $(0,78)$ e entre DCo e DDe $(0,72)$, o que significa que ovelhas com maior comprimento corporal tendem a ter perímetros torácicos maiores, e animais com maior diâmetro bi-costal terão maior diâmetro dorso-esternal.

Estimativas similares foram registradas em ovelhas Texel por Oliveira et al. (2007) e ovinos da raça Cabugi e Morada Nova por Silva et al. (2007), sendo os dados sobre o desenvolvimento da caixa torácica importantes por estar relacionados diretamente com o consumo de alimento, a capacidade respiratória e também digestiva dos ovinos (Santana et al., 2001).

Seria interessante em futuras pesquisas adicionar a mensuração do peso corporal e estabelecer correlações com as demais variáveis fenotípicas, buscando maior acurácia na estimação do desempenho animal, o que serviria na caracterização e melhoramento destes rebanhos locais.

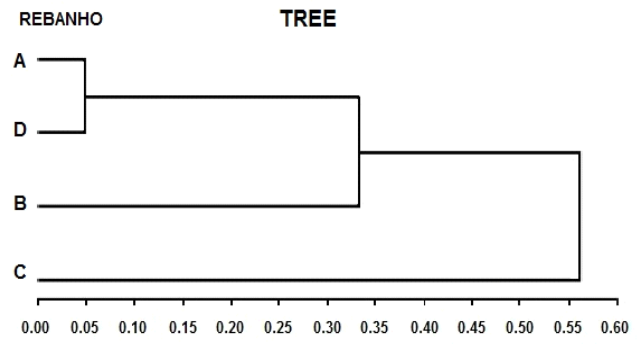

Figura 5. Dendrograma da análise de cluster. (Dendrogram from cluster analysis).

Ao invés de considerar os ovinos dos Humedales de Ite como um único grupo, é possível explorar a variação morfométrica existente, já que cada criatório apresentou uma especificidade.

Devem-se programar estratégias entre os criadores, autoridades públicas e instituições de pesquisa e desenvolvimento, visando identificar e organizar os grupos genéticos existentes, tendo essa alternativa por meio dos marcadores moleculares (Paiva e McManus, 2008). Assim se resgataria os ovinos crioulos em núcleos de conservação, e exploraria os animais

Tabela VI. Estimativas de correlação entre medidas morfométricas de ovelhas adultas criadas nos Humedales de Ite. (Estimates of correlation between morphometric measurements of adult ewes farmed in the Ite Wetlands).

\begin{tabular}{|c|c|c|c|c|c|c|c|c|c|c|c|c|}
\hline & $\mathrm{LCa}$ & CCo & $\mathrm{ACe}$ & PTo & $\mathrm{PCa}$ & DCo & DDe & $A G a$ & CGa & LGa & LAn & LIs \\
\hline $\mathrm{CCa}$ & $0,43^{* * *}$ & $0,64^{\star * \star}$ & $0,37^{\star *}$ & $0,63^{\star \star \star}$ & ${ }^{*} 0,44^{* \star *}$ & $0,54^{\star * *}$ & $0,51^{* \star *}$ & ${ }^{*} 0,35^{\star *}$ & $0,50^{\star * *}$ & ${ }^{*} 0,59^{\star \star \star *}$ & ${ }^{*} 0,21^{*}$ & $0,21^{*}$ \\
\hline LCa & & $0,24^{*}$ & $0,30^{*}$ & $0,25^{*}$ & $0,27^{\star}$ & $0,26^{*}$ & $0,24^{*}$ & $0,27^{\star}$ & $0,50^{* \star *}$ & ${ }^{*} 0,20$ & 0,13 & $0,23^{*}$ \\
\hline CCo & & & $0,45^{* * *}$ & $0,78^{\star * *}$ & ${ }^{*} 0,43^{* * *}$ & $0,67^{* * *}$ & $0,58^{* * *}$ & ${ }^{*} 0,32^{*}$ & $0,48^{* * *}$ & ${ }^{*} 0,50^{* * *}$ & $0,29^{*}$ & $0,24^{*}$ \\
\hline $\mathrm{ACe}$ & & & & $0,34^{* *}$ & 0,16 & $0,43^{* * *}$ & $0,51^{* * *}$ & ${ }^{*} 0,50^{\star \star \star}$ & $0,30^{*}$ & $0,32^{*}$ & $0,22^{*}$ & $0,36^{* *}$ \\
\hline PTo & & & & & $0,45^{\star * *}$ & $0,68^{* * *}$ & $0,64^{* * *}$ & ${ }^{*} 0,31^{*}$ & $0,55^{* * *}$ & ${ }^{*} 0,45^{\star * *}$ & $0,33^{* *}$ & $0,21^{*}$ \\
\hline $\mathrm{PCa}$ & & & & & & $0,45^{\text {***}}$ & $0,48^{* * *}$ & ${ }^{*} 0,21^{*}$ & $0,28^{*}$ & $0,30^{*}$ & $0,21^{*}$ & $0,31^{*}$ \\
\hline DCo & & & & & & & $0,72^{\star * *}$ & ${ }^{*} 0,30^{*}$ & $0,59^{\star * *}$ & ${ }^{*} 0,47^{\star \star *}$ & ${ }^{*} 0,35^{\star *}$ & $0,21^{*}$ \\
\hline DDe & & & & & & & & $0,40^{* * *}$ & $0,41^{* * *}$ & ${ }^{*} 0,46^{* * *}$ & $0,37^{* *}$ & $0,29^{*}$ \\
\hline AGa & & & & & & & & & $0,31^{*}$ & $0,24^{*}$ & $0,24^{*}$ & $0,33^{* *}$ \\
\hline CGa & & & & & & & & & & $0,25^{*}$ & $0,32^{*}$ & $0,32^{*}$ \\
\hline LGa & & & & & & & & & & & 0,10 & $0,24^{*}$ \\
\hline LAn & & & & & & & & & & & & $0,22^{*}$ \\
\hline
\end{tabular}

${ }^{*} \mathrm{p}<0,05 ;{ }^{* *} \mathrm{p}<0,01 ;{ }^{* * *} \mathrm{p}<0,001$. 


\section{MONTESINOS, SILVA, LOPES, FIORAVANTI, MCMANUS E SERENO}

mestiços para aproveitar possíveis vantagens zootécnicas produto da heterose, lembrando que cada biótipo possui exigências diferentes, as quais devem estar de acordo com a realidade dos criadores (Chacón et al., 2011).

\section{CONCLUSÕES}

As ovelhas criadas nos Humedales de Ite são menores do que raças especializadas, mas possuem tendência para aptidão

\section{BIBLIOGRAFIA}

Alencastre, R. y Gómez, N. 2005. Comportamiento reproductivo del ovino criollo en el altiplano peruano. Arch Zootec, 54: 541-544.

Álvarez, S., Fresno, M., Capote, J., Delgado, J.V. y Barba, C. 2000. Estudio para la caracterización de la raza ovina Canaria. Arch Zootec, 49: 209-215.

Alves, A.G.C., Pires, D.A.F. e Ribeiro, M.N. 2010. Conhecimento local e produção animal: uma perspectiva baseada na Etnozootecnia. Arch Zootec, 59: 45-56.

Bianchini, E., McManus, C., Lucci, C.M., Fernandes, M.C.B., Prescott, E., Mariante, A.S. e Egito, A.A. 2006. Características corporais associadas com a adaptação ao calor em bovinos naturalizados brasileiros. Pesqui Agropecu Bras, 41: 1443-1448.

Bravo, S. y Sepúlveda, N. 2010. Índices zoométricos en ovejas criollas Araucanas. Int J Morphol, 28: 489-495.

Carneiro, H.,Louvandini, H., Paiva, S.R., Macedo, F., Mernies, B. and McManus, C. 2010. Morphological characterization of sheep breeds in Brazil, Uruguay and Colombia. Small Ruminant Res, 94: 58-65.

Cartwright, T.C. 1979. Size as a component of beef production efficiency: cow-calf production. J Anim Sci, 48: 974-980.

Chacón, E., Macedo, F., Velásquez, F., Paiva, S.R., Pineda, E. and McManus, C. 2011. Morphological measurements and body indices for Cuban Creole goats and their crossbreds. Rev Bras Zootecn, 40: 1671-1679.

Dossa, L.H., Wollny, C. and Gauly, M. 2007. Spatial de corte. Ocorre variação entre rebanhos no tamanho corporal, verificada pela existência de diferentes biótipos, que podem ser determinados pelo local de criação, manejo praticado e aparentes graus de miscigenação com raça exótica. É necessário caracterizar e organizar o fluxo gênico, tanto dos ovinos crioulos como mestiços, possibilitando maiores subsídios para a conservação dos recursos genéticos locais e o desenvolvimento sustentável da ovinocultura tradicional no sul do Peru.

variation in goat populations from Benin as revealed by multivariate analysis of morphological traits. Small Ruminant Res, 73: 150-159.

Egito, A.A., Mariante, A.S. e Albuquerque, M.S.M. 2002. Programa brasileiro de conservação de recursos genéticos animais. Arch Zootec, 51: 39-52.

Flores, D. 2008. Crianza de ovinos. http:// www.articulo.org/articulo/3625/crianza de_ovinos_i.html (25/04/2011).

Fulcrand, B. 2005. Melhorando o desempenho de raças locais de ovelhas. Agriculturas, 2: 16-19. Herrera, M., Rodero, E., Gutierrez, M.J., Peña, F. and Rodero, J.M. 1996. Application of multifactorial discriminant analysis in the morphostructural differentiation of Andalusian caprine breeds. Small Ruminant Res, 22: 3947.

INIA. 2003. Instituto Nacional de Innovación Agraria. Programa Nacional de Investigación Ovinos. Ministerio de Agricultura del Perú. http:// www.inia.gob.pe (04/04/2011).

Mariante, A.S. e Cavalcante, N. 2006. Animais do descobrimento: Raças domésticas da historia do Brasil. 2ª ed. Embrapa. Brasília. 274 pp.

McManus, C.M., Santos, S.A., Silva, J.A., Louvandini, H., Abreu, U.G.P., Sereno, J.R.B. and Mariante, A.S. 2008. Body indices for the Pantaneiro horse. Braz J Vet Res Anim Sci, 45: 362-370.

Miserani, M.G., McManus, C., Santos, S.A., Silva, J.A., Mariante, A.S. e Abreu, U.G.P. 2002. Avaliação dos fatores que influem nas medidas lineares do cavalo Pantaneiro. Rev Bras

Archivos de zootecnia vol. 61, núm. 236, p. 514. 


\section{CARACTERIZAÇÃO FENOTÍPICA DE OVELHAS DOS HUMEDALES DE ITE, PERU}

Zootecn, 31: 335-341.

Oliveira, M.F., Ojeda Filho, S.C.F., Hernández, I., Leite, L., Souza, J.C., Abreu, U.G.P. e Sereno, J.R.B. 2007. Avaliação fenotípica de ovelhas da raça Texel criadas na parte alta do Pantanal. $B o l$ Pesqui Desenvolv, 76. http://www.cpap. embrapa.br/publicacoes/online/BP76.pdf (23/ 12/2010).

Pacheco, A. e Quirino, C.R. 2008. Estudo das características de crescimento em ovinos. Pubvet. http://www.pubvet.com. br/artigos_ det.asp?artigo=293 (10/03/2011).

Paiva, S.R. e McManus, C. 2008. Uso de marcadores moleculares como ferramenta adicional para conservação e melhoramento da raça Santa Inês. Em: Anais V Congresso Nordestino de Produção Animal. http://www.alice.cnptia.embrapa. br/bitstream/doc/894697 (20/01/2012).

Primo, A.T. 1992. El ganado bovino ibérico en las Américas: 500 años después. Arch Zootec, 41: 421-432.

Riva, J., Rizzi, R., Marelli, S. and Cavalchini, L.G. 2004. Body measurements in Bergamasca sheep. Small Ruminant Res, 55: 221-227.

Robles, W.L. 2009. Censo poblacional de rumiantes menores del distrito de Ite. Informe de actualización de la línea base del proyecto Mejoramiento de la crianza de cabras para la producción de leche y carne. Municipalidad Distrital de Ite. http://www.muniite.gob.pe/mdi/ (25/08/ 2009).
Rodríguez, P.L., Tovar, J.J., Rota, A.M., Rojas, A. y Martín, L. 1990. El exterior de la cabra Verata. Arch Zootec, 39: 43-57.

Rodríguez, F.P.C., Genís, J.M.C., Guerrero, J.L.G., Pertínez, M.D., Guerrero, Y.M., Aldea, M.J.A. y Redondo, P.G. 2003. Bases de la producción animal. $1^{\text {a }}$ ed. US/UCO. Sevilla. 516 pp.

Salako, A.E. 2006. Application of morphological indices in the assessment of type and function in sheep. Int J Morphol, 24: 13-18.

Santana, A.F., Costa, G.B. e Fonseca, L.S. 2001. Correlações entre peso e medidas corporais em ovinos jovens da raça Santa Inês. Rev Bras Saúde Prod Anim, 1: 74-77.

SAS. 2001. User's guide for windows environment 9.1.3 ed. SAS Institute Inc. Cary, N.C.

Silva, M.C. 2011. Estudo morfométrico de ovinos da raça Crioula Lanada no sul do Brasil: um subsídio para a conservação in situ. Dissertação de Mestrado. Universidade Federal de Goiás. Escola de Medicina Veterinária e Zootecnia, Goiânia. 86 pp.

Silva, N.V., Fraga, A.B., Filho, J.T.A., Neto, C.C.C., Silva, F.L., Costa, P.P.S. e Lira Junior, W.B. 2007. Caracterização Morfométrica de ovinos deslanados Cabugi e Morada Nova. Rev Cient Prod Anim, 9: 65-75.

Vizcarra, J.K. 2008. Los Humedales de Ite: Um Potencial Ecoturístico. http://www.darwinnet. org/docs/MANUAL_GUIA_HUMEDALES_ITE. pdf $(11 / 06 / 2011)$ 